\title{
The Schumann Resonances
}

\author{
R. K. Cole, Jr. \\ Stanford Research Institute, Menlo Park, Calif.
}

(Received January 27, 1965; revised May 10, 1965)

\begin{abstract}
The resonances of the earth ionosphere cavity are considered directly as a cavity problem rather than as a limiting case of propagation in a quasi-waveguide with an arbitrary (spherically symmetric) conductivity profile. By using an approximation to a theoretically derived profile, values for the frequencies and $Q$ factors of the lower resonances are computed which compare with experimental results as well as or better than the values derived from previous models. The effects of small changes in the profile are also considered. It is shown that ionic conduction in the lower atmosphere should not be neglected and that the "knee" in the conductivity profile where the transition from ionic to electronic conductivity occurs has a significant, and previously unrecognized, effect, particularly on the $Q$ factors of the resonant modes.
\end{abstract}

\section{Introduction}

The existence of resonances of the cavity between the earth and the lower edge of the ionosphere and their excitation by lightning flashes was predicted by Schumann [1952]. Experimental measurements have amply confirmed Schumann's prediction, and the resonant frequencies and $Q$ factors have been determined [Balser and Wagner, 1960]. The "quality factor," $Q$, is defined as $2 \pi$ times the ratio of the stored energy to the energy loss per cycle; it is a measure of the sharpness of the resonance. Theoretical calculations have been performed under various simplifying assumptions [e.g., see Schumann, 1952; Wait, 1964; Galejs, 196la, b, and 1962; Chapman and Jones, 1964; Jones, 1964]; many of these give reasonable overall agreement with experiment, but none is entirely satisfactory. The most successful models tend to be somewhat artificial and to show great sensitivity to artificially introduced parameters. Careful adjustment of parameters has been particularly necessary to reproduce the observed increase of $Q$ with increasing frequency.

It is conventional to approach the resonances as limiting cases of propagation in the quasi-waveguide between earth and ionosphere and to express the properties of the boundaries by surface impedances. The models that give the best results consider a uniformly conducting earth and either an exponential ionospheric conductivity profile, sharply bounded at some (often frequency-dependent) critical height [e.g., see Galejs, 1961a, b 1962], or an ionosphere constructed of several homogeneous layers [e.g., see Jones, 1964]. The effects of finite conductivity below the ionosphere may be treated by a later perturbation calculation.

Treatment of the problem explicitly as a resonant cavity and use of a smooth conductivity-height profile to eliminate the nonphysical surface charge at the lower "edge" of the ionosphere should simplify the analysis and produce better results. This has been done by using a conductivity profile recently derived theoretically from fundamental considerations by Cole and Pierce [1965].

\section{Differential Equations}

Maxwell's equations and the constitutive relations in mks units are

$$
\begin{array}{lll}
\nabla \times \mathbf{H}=\mathbf{J}+\frac{\partial}{\partial t} \mathbf{D} & \mathbf{D}=K \epsilon_{0} \mathbf{E} \\
\nabla \times \mathbf{E}+\frac{\partial}{\partial t} \mathbf{B}=0 & \mathbf{B}=\mu_{0} \mathbf{H} \\
\nabla \cdot \mathbf{D}=\rho & \mathbf{J}=\sigma \mathbf{E} \\
\nabla \cdot \mathbf{B}=0 . &
\end{array}
$$

Here $\mathbf{H}$ is the magnetic field, $\mathbf{E}$ the electric field, $\mathbf{B}$ the magnetic flux density, $\mathbf{D}$ the displacement, $\mathbf{J}$ the current density, and $\rho$ the charge density. The permittivity and permeability of free space are denoted by $\epsilon_{0}$ and $\mu_{0}$, respectively; $K$ is the local dielectric constant and $\sigma$ the conductivity.

The wave equation in $\mathbf{H}$ is derived in the usual way by assuming a universal exp $(i \omega t)$ time dependence, where $\omega$ may be complex, taking the curl of the $\nabla \times \mathbf{H}$ equation, and eliminating $\mathbf{B}, \mathbf{E}$, and $\mathbf{D}$. We find

$\nabla^{2} \mathbf{H}+\mu_{0} K \epsilon_{0} \omega^{2} \mathbf{H}-i \mu_{0} \sigma \omega \mathbf{H}$

$$
+\frac{\nabla\left(\sigma+i K \epsilon_{0} \omega\right) \times(\nabla \times \mathbf{H})}{\sigma+i K \epsilon_{0} \omega}=0 .
$$
Now, letting $\mu_{0} \epsilon_{0}=\frac{1}{c^{2}}, k=\frac{\omega}{c}$, and $\eta=\frac{\sigma}{\epsilon_{0} c}$, the equa-
tion becomes

$$
\left[\nabla^{2}+K k^{2}-i k \eta+\frac{\nabla(\eta+i K k) \times \nabla \times}{\eta+i K k}\right] \mathbf{H}=0 .
$$


For vertical polarization (the conventional case), $\mathbf{H} \cdot \mathbf{r}=0$, where $\mathbf{r}$ is the radius vector from the center of the earth, and, since $\nabla \cdot \mathbf{H}=0$, we may expand $\mathbf{H}$ in spherical coordinates as

$$
\mathbf{H}=\sum_{l m} \frac{f_{l m}(r)}{r} \mathbf{X}_{l}^{m}(\theta, \varphi),
$$

where $\mathbf{X}_{l}^{m}(\theta, \varphi)=\frac{1}{\sqrt{l(l+1)}} \mathbf{r} \times \nabla Y_{l}^{m}(\theta, \varphi)$, and the $Y_{l}^{m}(\theta, \varphi)$ are the ordinary spherical harmonics.

Substitution of this expansion into the differential equation, with the assumption that $\eta$ and $K$ are functions of $r$ only, gives the differential equation for the

$$
\begin{aligned}
& \text { radial function } f_{l m}(r) \\
& \left.\qquad \frac{d^{2}}{d r^{2}}-\frac{l(l+1)}{r^{2}}+K k^{2}-i k \eta-\frac{\frac{d}{d r}(\eta+i K k)}{\eta+i K k} \frac{d}{d r}\right] f_{l}(r)=0,
\end{aligned}
$$

where " $m$ " has been suppressed because it does not enter the operator. The necessary boundary conditions are that the energy flow should be outward from the cavity, downward into the earth, upward into the ionosphere.

If we further specialize to the case $\eta=\eta_{e}$, a constant, for $r<a$, the boundary condition at $r=a$ may be made explicit in simple form.

For $r<a$, within the earth, $\eta \sim 10^{5} \mathrm{~km}^{-1}, \mathrm{r} \sim 6 \times 10^{4}$ $\mathrm{km}$, and $|k| \sim 10^{-4} \mathrm{~km}^{-1}$ at ELF, where $|k|$ is the magnitude of (complex) $k$, so the leading terms of the differential equation are

$$
\left[\frac{d^{2}}{d r^{2}}-i k \eta_{e}\right] f(r)=0 .
$$

The appropriate solution, corresponding to a downward wave that decays with time, is

$$
f(r)=A \exp \left[\left(i k \eta_{e}\right)^{1 / 2} r\right]
$$

where the square root is chosen to be in the first quadrant for $k$ in the first quadrant.

Just below the earth's surface,

$$
\frac{d}{d r} f\left(a_{-}\right)=\left(i k \eta_{e}\right)^{1 / 2} f\left(a_{-}\right) .
$$

Assuming the earth-air transition to be sufficiently abrupt that the $\frac{d}{d r}(\eta+i K k)$ term in the radial equation predominates, we may write

$$
\frac{d}{d r}\left(\frac{d f}{d r}\right) / \frac{d f}{d r}=\frac{d}{d r}(\eta+i K k) /(\eta+i K k)
$$

so that

$$
\frac{d f}{d r}\left(a_{+}\right)=\frac{\eta\left(a_{+}\right)+i k}{\eta_{e}+i K_{e} k} \frac{d f}{d r}\left(a_{-}\right) \approx \frac{\eta\left(a_{+}\right)+i k}{\eta_{e}} \frac{d f}{d r}\left(a_{-}\right) .
$$

Choosing $A$ so that $f\left(a_{-}\right)=1$, we have the initial conditions at $r=a_{+}$

$$
\begin{aligned}
f\left(a_{+}\right) & =1 \\
f^{\prime}\left(a_{+}\right) & =\left(\eta\left(a_{+}\right)+i k\right)\left(i k / \eta_{e}\right)^{1 / 2} .
\end{aligned}
$$

The boundary condition for large $r$ may be expressed by requiring that the outward radial component of the Poynting vector, which may be written as

$S_{r}=\left(\mathbf{E} \times \mathbf{H}^{*}\right)_{r}$

$$
\begin{aligned}
\sim-[(\eta-\operatorname{Im} k) & \left(\operatorname{Re} f \operatorname{Re} f^{\prime}+\operatorname{Im} f \operatorname{Im} f^{\prime}\right) \\
+ & \operatorname{Re} k\left(\operatorname{Re} f \operatorname{Im} f^{\prime}-\operatorname{Im} f \operatorname{Re} f^{\prime}\right),
\end{aligned}
$$

remain positive. Here ${ }^{*}$ denotes complex conjugation, Re and Im denote real and imaginary part, respectively, and $f^{\prime}=\frac{d f}{d r}$.

In the frequency range, $\sim 7$ to $100 \mathrm{c} / \mathrm{s}$, appropriate to the lower Schumann resonances, $|k|$ and $1 / r$ are of comparable magnitude. At low altitudes, $\eta<<|k|$; at high altitudes, $\eta>>|k|$. For likely conductivity profiles, equality occurs at heights of 50 to $60 \mathrm{~km}$. The differential equation is explicitly soluble in these limits, if we assume an exponential profile, $\eta_{0} \exp (r / L)$ for $\eta$ at high altitudes. The solutions are

$$
\begin{gathered}
\eta<<|k| \quad\left(d^{2} / d r^{2}+k^{2}\right) f=0 \\
f=A \exp (i k r)+B \exp (-i k r) \\
\eta>>|k| \quad\left(d^{2} / d r^{2}-i k \eta_{0} \exp (r / L)-1 / L d / d r\right) f=0 \\
f=\exp (r / 2 L)\left[C H _ { 1 } ^ { ( 1 ) } \left(2 L\left(-i k \eta_{0}\right)^{1 / 2} \exp (r / 2 L)\right.\right. \\
\left.+D H_{1}^{(2)}\left(2 L\left(-i k \eta_{0}\right)^{1 / 2} \exp (r / 2 L)\right)\right]
\end{gathered}
$$

where $H_{1}^{(1)}$ and $H_{1}^{(2)}$ are the Hankel functions of order one of the first and second kind. If the square root is taken in the upper half plane, $H_{1}^{(1)}\left(H_{1}^{(2)}\right)$ represents the solution that is regular (irregular) at infinity. Only the first is appropriate to this problem; the second represents incident energy. A "matching" of the asymptotic solutions to find the eigenvalue $k$, and thus resonant frequency and $Q$, would be highly inaccurate; because both the regular and irregular solutions are constant to first order at the lower end of their domain of applicability, the transition region where $\eta \sim|k|$ is of great importance.

For any $k$ a solution - which does not in general satisfy the boundary condition at large $r$-may be found by numerical integration of the radial differential equation, starting from the known boundary condition at $r=a_{+}$. For $k$ not an eigenvalue, $S_{r}$. will become negative above some height; an inward flux of energy is necessary to support the imposed variation of $\mathbf{H}$ with $\varphi, \theta$, and $t$. By repeated integrations with successive refinements of the trial value of $k$, systematically chosen to maximize the height at which $S_{r}$ becomes negative, we may approximate the eigenvalue $k_{i}$ to any desired degree of accuracy. Restated, the height $h_{c}$ at which $S_{r}$ becomes negative, considered as a function of complex $k$, has a pole at the eigenvalue $\mathrm{kil}$. We are finding this pole by a simple ascent method. 
TABLE 1. Frequencies and Q factors of the cavity modes

\begin{tabular}{|c|c|c|c|c|c|c|}
\hline & $l=1$ & $l=2$ & $l=3$ & $l=4$ & $l=5$ & Profile \\
\hline $\begin{array}{l}\text { Measured } \\
\quad(1)\end{array}$ & $\begin{aligned} f & =7.8 \\
Q & =4.0\end{aligned}$ & $\begin{array}{r}14.1 \\
4.5\end{array}$ & $\begin{array}{r}20.3 \\
5.0\end{array}$ & $\begin{array}{r}26.3 \\
5.5\end{array}$ & $\begin{array}{r}32.5 \\
6.0\end{array}$ & \\
\hline Lossless & $f=10.6$ & 18.3 & 25.9 & 33.5 & 41.1 & \\
\hline $\begin{array}{l}\text { Constant } h^{\prime} \\
\quad(2)\end{array}$ & $\begin{aligned} f & =8.3 \\
Q & =7.6\end{aligned}$ & 14.2 & 20.0 & 25.8 & $\begin{array}{l}31.7 \\
15.8\end{array}$ & $\begin{array}{rl}\eta=1.6 \times 10^{-10} \exp (h / 3.25) \mathrm{km}^{-1} h & >h^{\prime}=50 \mathrm{~km} \\
0 & 0<h<h^{\prime}\end{array}$ \\
\hline $\begin{array}{c}\sigma\left(h^{\prime}\right)=\omega \epsilon_{0} \\
(2)\end{array}$ & $\begin{array}{r}f=8.0 \\
Q=3.8\end{array}$ & 13.8 & 19.5 & 25.4 & $\begin{array}{l}31.3 \\
11.8\end{array}$ & $\eta=1.6 \times 10^{-10} \exp (h / 3.25) \mathrm{km}^{-1} h>h^{\prime}$ \\
\hline $\begin{array}{l}\text { "Average" } \\
\text { (3) }\end{array}$ & $\begin{array}{r}f=8.05 \\
\text { " } Q=6.75 \\
\text { a c c } 4.5\end{array}$ & 14.25 & 20.5 & 26.8 & $\begin{array}{l}\text { if } 5.7 \\
\text { b c } 4.7\end{array}$ & $\begin{array}{l}\eta=1.6 \times 10^{-10} \exp (h / 3.25) \mathrm{km}^{-1} h^{\prime}=45 \mathrm{~km} \text { (Day) } \\
\eta=8.1 \times 10^{-16} \exp (h / 2.25) \mathrm{km}^{-1} h^{\prime}=60 \mathrm{~km} \text { (Night) }\end{array}$ \\
\hline $\begin{array}{l}\text { Two Layer } \\
\text { (4) }\end{array}$ & $\begin{aligned} f & =7.90 \\
Q & =4.7\end{aligned}$ & $\begin{array}{l}14.13 \\
6.0\end{array}$ & $\begin{array}{r}20.3 \\
6.9\end{array}$ & $\begin{array}{r}26.4 \\
7.5\end{array}$ & $\begin{array}{r}32.6 \\
8.2\end{array}$ & $\eta=\left\{\begin{array}{lrl}0 & 0<h<70 \mathrm{~km} \\
1 \times 10^{4} \mathrm{~km}^{-1} & 70<h<100 \mathrm{~km} \\
4.9 \times 10^{5} \mathrm{~km}^{-1} & h>100\end{array}\right.$ \\
\hline I & $\begin{aligned} f & =7.43 \\
Q & =4.1\end{aligned}$ & & $\begin{array}{r}19.4 \\
4.7\end{array}$ & & $\begin{array}{r}31.5 \\
5.1\end{array}$ & $\begin{aligned} \eta & =7.5 \times 10^{-8} \exp (h / 6.4)+2.3 \times 10^{-12} \exp \\
\eta_{r} & =10^{5} \mathrm{~km}^{-1} \quad(h / 3.0) \mathrm{km}^{-1}\end{aligned}$ \\
\hline & & & $\begin{array}{l}f \pm 0.2 \% \\
Q \pm 0.5 \%\end{array}$ & & & Same, $\eta_{e}=10^{6}, 10^{4}$ \\
\hline II & $\begin{aligned} f & =7.55 \\
Q & =4.5\end{aligned}$ & & $\begin{array}{r}19.6 \\
5.3\end{array}$ & & $\begin{array}{r}31.9 \\
5.6\end{array}$ & $\begin{aligned} \eta & =5.0 \times 10^{-8} \exp (h / 6.4)+2.3 \times 10^{-12} \exp \\
\eta_{c} & =10^{5} \mathrm{~km}^{-1} \quad(h / 3.0) \mathrm{km}^{-1}\end{aligned}$ \\
\hline III & $\begin{aligned} f & =7.71 \\
Q & =4.6\end{aligned}$ & $\begin{array}{r}13.9 \\
5.1\end{array}$ & $\begin{array}{r}20.0 \\
5.4\end{array}$ & $\begin{array}{r}26.2 \\
5.6\end{array}$ & $\begin{array}{r}32.4 \\
5.8\end{array}$ & $\begin{array}{c}\eta=5.0 \times 10^{-8} \exp (h / 6.4)+2.2 \times 10^{-13} \operatorname{exr} \\
\eta_{e}=10^{5} \mathrm{~km}^{-1} \quad(h / 2.7) \mathrm{km}^{-1}\end{array}$ \\
\hline
\end{tabular}

(1) Balser and Wagner [1960].

(2) Galejs [1961b].

(3) Galejs [1962].

(4) Jones [1964].

At $f=10$.

") $f=30$.

"With "cosmic ray" ionization, $\eta$ exponential below $h^{\prime} \eta=3.76 \times 10^{-8} \mathrm{~km}^{-1}$ at $h=0$, and continuous at $h^{\prime}$

A typical modeform is shown in figure 2. Although drawn for the $l=1$ mode with profile $\mathrm{I}$, these profiles are all but indistinguishable from those for other modes and conductivity profiles. Energy and loss density profiles derived from this are given in figure 3 . The energy densities are $\frac{1}{2} \mu_{0} \mathbf{H} \cdot \mathbf{H}^{*}$ and $\frac{1}{2} \epsilon_{0} \mathbf{E} \cdot \mathbf{E}^{*}$; the loss density is $\left|\mathbf{E} \cdot \mathbf{J}^{*}\right|$. Note that the loss density is significant only near the height where $\eta=|k|$ (for $\operatorname{Im} k<<\operatorname{Re} k$ this is $\left.\omega=\sigma / \epsilon_{0}\right)$.

\section{Importance of the Knee of the Conductivity Profile}

From the definition of $Q$ as $2 \pi$ times the ratio of stored energy to energy loss per cycle, we have

$$
Q=\frac{\omega\left[\epsilon_{0} \int \mathbf{E} \cdot \mathbf{E}^{*} d V+\mu_{0} \int \mathbf{H} \cdot \mathbf{H}^{*} d V\right]}{2 \int \mathbf{E} \cdot \mathbf{J}^{*} d V}
$$

In the cavity, $r \approx a$, the earth radius, and

$$
\begin{gathered}
H \approx \frac{f}{a} \mathbf{X}_{l}=f \mid a(l(l-1))^{-1 / 2} \frac{\partial}{\partial \theta} Y_{l}(\theta, \varphi) \hat{e}_{\theta} \\
E_{r} \approx \frac{1}{a} f \mid a\left(\sigma+i \epsilon_{0} \omega\right)^{-1}\left(\frac{\partial^{2}}{\partial \theta^{2}}+\operatorname{ctn} \theta \frac{\partial}{\partial \theta}\right)(l(l+1))^{-1 / 2} Y_{l}(\theta, \varphi) \\
=\frac{c \mu}{a^{2}} \frac{f}{\eta+i k}(l(l+1))^{1 / 2} Y_{l}(\theta, \varphi)
\end{gathered}
$$

$\mathbf{E} \approx E_{r} \hat{e}_{r}$ at low altitudes

$$
J_{r}=\sigma E_{r} \approx \frac{\eta f}{a^{2}(\eta+i k)}(l(l+1))^{1 / 2} Y_{l}(\theta, \varphi) .
$$

The spherical harmonics are normalized to

$$
\int \mathbf{X}_{l}^{*} \cdot \mathbf{X}_{l} d \Omega=\int Y_{l}^{*} Y_{l} d \Omega=1
$$

so that (neglecting field penetration into the earth)

$$
Q \approx \frac{\omega}{2 c} \frac{l(l+1) \int_{0}^{\infty}|f|^{2} /\left(\eta^{2}+|k|^{2}\right) d h+a^{2} \int_{0}^{\infty}|f|^{2} d h}{l(l+1) \int_{0}^{\infty}|f|^{2} /\left(\eta^{2}+|k|^{2}\right) d h} .
$$

Now if we approximate $\eta$ by $\eta_{0} \exp (r / L)$ near $h_{0}$, where $\eta=|k|$, and observe that $|f| \approx 1$ for $0<h<h^{*}$, and $f \approx 0$ elsewhere, we have upon performing the integrals

$$
Q_{l} \approx \frac{1}{\pi L_{l}}\left(h_{o l}+\frac{\left(\operatorname{Re} k_{l} a\right)^{2}}{l(l+1)} h_{l}^{*}\right),
$$

where $\operatorname{Re} k \approx|k|$ has been used.

Note in figure 1 that the points where $|k|=\eta$ for $l=1$ to $5\left(|k| \sim 2\right.$ to $\left.7 \times 10^{-4} \mathrm{~km}^{-1}\right)$ lie on the knee of the curve. This has two effects: the effective value of $L$ decreases as $l$ increases, and the increase in $h_{0}$ as $l$ increases is greater than it would be for a single exponential fitting the upper portion of the conductivity profile. Both tend to produce an increase in $Q$ with increasing $l$. Therefore neglect of ionic conductivity, which is responsible for the knee, is not justified. Treating it as a perturbation to compute corrections to the Qs, as has been done before [Galejs, 1962], is an 
